\title{
Philosophiques
}

\section{Les contributions de la science à la philosophie au $20^{\mathrm{e}}$ siècle}

\section{Larkin Kerwin}

Volume 16, numéro 1, printemps 1989

URI : https://id.erudit.org/iderudit/027067ar

DOI : https://doi.org/10.7202/027067ar

Aller au sommaire du numéro

Éditeur(s)

Société de philosophie du Québec

ISSN

0316-2923 (imprimé)

1492-1391 (numérique)

Découvrir la revue

Citer cet article

Kerwin, L. (1989). Les contributions de la science à la philosophie au $20^{\mathrm{e}}$ siècle. Philosophiques, 16(1), 149-161. https://doi.org/10.7202/027067ar d'utilisation que vous pouvez consulter en ligne.

https://apropos.erudit.org/fr/usagers/politique-dutilisation/ 


\title{
INTERVENTION
}

\author{
LES CONTRIBUTIONS \\ DE LA SCIENCE A LA PHILOSOPHIE \\ AU $20^{\mathrm{e}}$ SIĖCLE * \\ par Larkin Kerwin
}

\section{INTRODUCTION}

Je remercie les autorités de l'Université Laval qui m'accordent le privilège de présenter la première Conférence Rasetti - 1988.

Le professeur Franco Rasetti est un des grands intellectuels à avoir séjourné à l'Université Laval. L'ambiance qu'il a créée et les principes dont il a imprégné le Département de physique, qu'il a fondé, sont toujours d'actualité. Je fus son dernier étudiant au doctorat à Laval, et je me souviens très bien de cette ambiance ainsi que de l'énergie immense qu'il appliquait à toute tâche et à toute discussion. Bien que connu surtout comme physicien, il était, à vrai dire, un généraliste qui était à l'aise pour discuter aussi bien de la chimie, de la géologie, ou de la paléontologie - discipline qu'il a maîtrisée d'ailleurs, au point d'en être une autorité internationale. La philosophie aussi attirait ses considérations et ses commentaires. À cette époque, Laval bénéficiait également de la présence du grand Charles De Koninck - un Thomiste des plus renommés. Rasetti et De Koninck figuraient alors ensemble lors de colloques et de tables rondes, nous émerveillant non seulement par leur

* Texre de la communication prononcée le 2 mai 1988, par le Dr Larkin Kerwin, alors président du Conseil National de Recherches du Canada, à l'occasion des Conférences Rasetti, pour le cinquantième anniversaire de la Faculté des Sciences et de Génie de l'Université Laval. Le Dr Kerwin est maintenant président de l'Agence spatiale canadienne. Merci au professeur R.J. SLOBODRIAN, du département de physique de cette Faculté, d'en avoir permis la publication dans Philosophiques. 
érudition et par leur sagesse, mais aussi par les panoramas intellectuels qu'ils nous déployaient - même si c'était avec des points de vue souvent fort dissemblables.

Rien de surprenant là : la philosophie naturelle ("physique ») et la philosophie ont été partenaires à travers l'histoire dans le tango téléologique qui a animé les générations et, en 1948, on voyait que Rasetti sentait les changements dans l'air au sujet des Grandes Idées. Ses travaux y contribuaient, comme nous allons le voir. Aujourd'hui, ces changements sont le sujet d'écrits innombrables, et il n'y a pas une semaine qui passe sans que de nouveaux articles et de nouveaux textes touchant la nouvelle philosophie voient le jour.

Comme dans ces écrits il s'agit souvent de la physique à la Rasetti, je me propose, ce soir, de faire le joint entre divers as pects de son œuvre et de ces idées nouvelles. Vous aurez alors un aperçu de cette évolution de la philosophie par un spectateur très intéressé par les effets de sa discipline sur la vie intellectuelle plus large.

\section{SCIENCE ET PHILOSOPHIE}

Ces deux activités intelligentes s'entremêlent depuis toujours.

La science:

une bonne description de la science est la suivante : une activité humaine qui cherche à déterminer la description de l'univers. Pour ce faire, la science procède par approximations successives qui approchent de façon asymptotique la Vérité.

La philosophie : il s'agit ici de l'étude rationnelle de la pensée humaine. Ceci nous donne un encadrement - un point de vue sur notre monde et sur nos vies. La philosophie imprègne alors notre vie sociale; les grands thèmes politiques, économiques, psychologiques et moraux y sont ancrés : l'ambiance de nos foyers, de nos villes, de nos médias, de nos gouvernements en est entachée et colorée. C'est la philosophie qui nous suggère que le monde est positif ou - malheureux. 
Le lien entre ces deux vastes édifices de la connaissance est intime.

\section{LA SCIENCE : LES DESCRIPTIONS}

Esquissons quelques descriptions de l'univers que les savants ont imaginées.

Il y avait celle des anciens grecs qui, observant la nature et cherchant ses facteurs essentiels, ont proposé que l'univers possédait quatre éléments : la terre, l'air, le feu et l'eau. Tout le reste était composé de ces constituantes. Ainsi, la parole était de l'air et du feu; la vapeur, de l'eau et de l'air ; l'or, de la terre et du feu. C'était un système de grande beauté et de simplicité. Il a légué à la science future la notion d'un ordre fondamental, et nous trouvons sa caractéristique principale dans le tableau des éléments de Mendeléev. Il a stimulé le développement du précurseur de la chimie - l'alchimie - alors que l'on se consacrait à trouver la bonne combinaison des quatre éléments pour produire de l'or.

Une autre description fut celle appelée mécanique. Pour elle, l'univers était composé de masses en mouvement. Ces mouvements étaient réglés par les trois lois du mouvement de Newton, et sa loi de la gravitation universelle. Que ce soit les déplacements de planètes, une chute de neige, un courant d'eau et les va-et-vient d'une balle de tennis, tout se résumait par ces quatre lois. C'était un chef-d'œuvre de simplicité, et une des descriptions les plus puissantes jamais composées - la plus grande partie du génie moderne y est encore basée, et ses notions de base feront toujours partie de notre héritage intellectuel.

Une troisième description s'appelle ondulatoire. Elle décrit tout un spectre de phénomènes - les micro-ondes, la lumière, les rayons-X, les rayons cosmiques, comme étant une vibration électromagnétique. Notre technologie moderne en regorge. Des extensions de cette description que sont la méca nique ondulatoire et l'électrodynamique quantique sont à la fine pointe de la science moderne, décrivant graduellement même les structures de la bio-technologie. Dans un sens, alors, nous sommes nous-mêmes tous composés d'ondes.

Mentionnons aussi la description atomique de l'univers. Selon cette dernière, rien dans le cosmos ne serait continu: tout 
serait plutôt granulaire, quantifié, atomique. La matière serait composée ultimement d'atomes, l'énergie serait une série de quanta, l'espace serait divisé en petites zones "permises" alors que d'autres seraient interdites. Même le temps, postule-t-on, serait atomique: des intervalles de durée, appelées "chronons", étant séparées par d'autres qui seraient non temporelles. La description atomique est une autre création intellectuelle puissante du $20^{e}$ siècle.

Ce qui est merveilleux dans ces descriptions acceptées par la science c'est la simplicité avec laquelle elles résument la complexité inestimable du cosmos. Plus encore, elles sont caractérisées par la beauté et l'élégance. En effet, la beauté, la simplicité et l'élégance sont les critères selon lesquels les scientifiques jugent leurs descriptions ou leurs théories. Et quoique basées sur les faits expérimentaux, ces théories sont avancées en bonne partie par l'intuition, poussées par la curiosité. Nous reconnaissons dans ces qualités les mêmes que celles qui marquent la musique et l'art - voire même la philosophie.

Ainsi, à travers l'histoire, la science nous a fourni des descriptions de l'univers, correspondant aux faits alors connus. Chaque description acceptée a légué quelque chose d'elle-même aux descriptions subséquentes. Et dans ces créations de l'esprit que sont les théories scientifiques, les philosophes ont souvent puisé des éléments de leurs considérations de l'état humain.

\section{LE LIEN}

D'ailleurs, les philosophes ont souvent été eux-mêmes des observateurs-interprètes de la nature. Démocrite, Aristote et toute une galaxie de penseurs grecs ont manifesté une curiosité marquée pour les faits de la science à leur portée. Leurs noms dénotent plusieurs des philosophies les plus respectées.

Un exemple marqué du lien entre la science et la philosophie nous vient des siècles récents. En effet, le début du $20^{e}$ siècle était caractérisé par la philosophie dite matérialiste. Nous disons encore que nous vivons dans un monde matérialiste.

Cet état de la pensée est sorti de la Renaissance. Les savants comme Bacon, Galilée et Newton avaient démontré, de façon 
incontestable alors, la nature mécanique du système solaire et son extension à l'ensemble des phénomènes observables. Le modèle mécanique était tout-puissant, et, selon cet aperçu, le tout était caractérisé par le déterminisme, l'absolu, l'inexorabilité des mouvements et de leurs suites. L'humanité y figurait pour peu de chose: déplacée comme centre de l'univers par les mécaniciens, elle occupait une place sans importance particulière et sans signification. Dans un cosmos éternel, immuable et déterminé, tout se réduisait à la mécanique. L'intelligence même était perçue comme une suite de réactions mécaniques et chimiques. La volonté libre n'avait pas de place; aucun destin n'était particulier ou notable.

Pendant trois siècles, cette ligne de pensée fut développée par les plus grands esprits: les Descartes, les Sartre, les Camus. En particulier, le problème de la connaissance du matériel par une intelligence matérielle semblait insoluble. «je pense, donc je suis» fut l'ultime refuge de Descartes devant le dilemme. Hegel proposa que la matière même ne fut que la créature d'une conscience matérielle; Freud a démontré la non réalité du non matériel tel que l'esthétique. En somme, dans l'univers matérialiste et mécanique, il n'y avait pas de place pour l'ésotérique, les qualités dites artistiques, les vertus, la charité, la compassion, une vie morale, Dieu. L'on niait tout cela en faveur de réactions moléculaires.

Citons quelques conclusions de ces philosophes.

Descartes écrivit: "Ni le beau, ni l'agréable signifie autre chose que l'attitude de notre jugement ».

Freud conclut: "L'bumanité doit se rendre compte de la nature complète de son désarroi et de son insignifiance dans la machinerie de l'univers».

Bertrand Russel dit: «L'bomme est le produit de causes qui n'ont pas de conscience des buts vers lesquels elles s'acbeminent; ses origines, sa croissance, ses espoirs et ses craintes, ses amours et ses croyances ne sont que le résultat de collisions accidentelles d'atomes... seulement dans le chantier de ces vérités, seulement sur la fondation d'un désespoir solide peut-on songer à créer une babitation pour l'âme».

Tout ceci est peut-être vrai, et certes était assis sur une base intellectuellement satisfaisante au début du siècle. Mais il reste que 
c'est un aperçu de l'univers et de l'humanité qui est sombre et qui semble pessimiste. Pourtant, nous y avons érigé une civilisation très puissante: le monde matérialiste du $20^{e}$ siècle, avec ses systèmes politiques et économiques, ses richesses et ses complexités, ses merveilles industrielles et sociales, ses deux guerres mondiales et son assaut sur l'environnement. Cette philosophie a évidemment déteint sur d'autres disciplines, par exemple, en psychologie, où nous avons vu émerger le comportementalisme de John Watson.

Mais depuis le début du siècle, il s'est passé plusieurs choses en science pour perturber tout cela.

Je vais en mentionner quatre.

\subsection{La Relativité}

Maintenant de conséquence même industrielle, la Relativité fut au début un sujet de discussions surtout intellectuelles.

Nous nous rappelons que la description mécanique du cosmos nous présentait un univers éternel, absolu et déterminé.

Or, au début du siècle, Albert Einstein nous proposa que l'espace et le temps n'étaient pas absolus, mais présentaient des aspects variables, qui, en plus, dépendaient de l'observateur. Des vérifications expérimentales ne tardaient pas à confirmer cette nouvelle idée fondamentale, ainsi que les conséquences plutôt inattendues qui en découlaient.

Parmi elles étaient la destruction de la notion de simultanéité ; la variation de la longueur et le ralentissement des horloges avec la vitesse, l'équivalence de la masse et de l'énergie; le paradoxe des jumeaux, et ainsi de suite. L'examen des effets de la Relativité qui opposent nos bons sens est encore un exercice délicieux et un régal pour l'intelligence.

Le tout a été confirmé et vérifié maintes fois par des expériences en physique et en astronomie, ainsi que par des applications industrielles telles que dans les ordinateurs et la construction des grands accélérateurs.

L'œuvre de Franco Rasetti contribua à l'évolution de cette théorie. Je me souviens de ses expérirences avec les rayons 
cosmiques, effectuées avec la collaboration de Claude Geoffrion, George Hall, Paul Koenig et Fernand Bonenfant, utilisant des détecteurs de fabrication maison, comme étaient tous les appareils du Département dans le temps.

Rasetti a mesuré la demi-vie des particules cosmiques particulières - les mésons, appelés dans ce temps-là les mésotrons. I] trouvait une différence selon que les particules étaient arrêtées après un passage dans le plomb, dans l'aluminium ou dans d'autres substances, dans lesquelles elles avaient des vitesses particulières, ce qui affectait leurs "horloges". Cela m'a toujours surpris que cette vérification de l'effet relativiste n'ait pas eu plus d'attention de la part des historiens de la science.

La Relativité posa alors deux problèmes pour la philosophie matérialiste.

Tout d'abord, l'univers ne serait pas absolu, l'espace et le temps seraient des dimensions du cosmos dont l'allure variait avec les circonstances.

En second lieu, ces circonstances concernaient l'observateur - l'humanité. Que l'horloge soit ralentie de tel ou tel montant, que la masse augmente de telle ou telle fraction, lequel des jumeaux vieillissait moins vite que l'autre: tout dépendait des circonstances de l'observateur, de sa vitesse relative et de sa proximité des masses. Et quelle que soit l'allure observée, chaque observateur avait raison.

Alors la véritable description de l'univers dépendait de l'homme.

Ainsi, l'homme, déplacé au centre de l'univers par les interprètes de Copernic, regagna un peu de son importance.

\subsection{La mécanique quantique}

Un deuxième événement d'importance dans le monde scientifico-philosophique fut la mise au point de la théorie de la mécanique quantique. Initiée par Planck de façon empirique, Einstein démontra son enracinement dans l'effet photoélectrique, tel qu'interprété par la relativité. Et puis, une galaxie des plus grands noms de la science - Bohr, Schroedinger, Heisenberg, 
Pauli et d'autres - l'élaboreront en mécanique ondulatoire et en électrodynamique quantique, la plus puissante idée théorique de notre génération.

Or, d'après la mécanique quantique, les états énergiques et même géométriques de la matière ne pouvaient varier continuellement, mais seulement par étapes, par quanta. Les états possibles étaient discrets. En plus, nous ne pouvions connaître avec certitude ces énergies ou ces positions, mais seulement avec une certaine probabilité. Cette dernière notion fut érigée en principe - le Principe d'incertitude, qui fixait l'erreur probable dans un paramètre, alors que l'observateur en mesurait un autre. Par conséquent, si l'on décidait d'effectuer une mesure de, disons, le temps d'émission d'un photon, son énergie serait affectée de façon indéterminée pour toujours. Alors, non seulement l'univers prendrait une allure qui dépendait des circonstances de l'observateur, mais il était affecté par ses décisions. Voilà un dur coup pour le déterminisme.

L'ceuvre de Rasetti porta beaucoup sur cette question. Avant tout, un spectroscopiste de réputation internationale, il mesura les spectres des atomes et des molécules, vérifia les états quantiques, confirma les transitions interdites, posant autant de difficultés au modèle mécanique de l'univers. Il expliqua l'évolution de la pensée déterministe ainsi : «Je peux parler de la position de la lune même si je ne la vois pas, car je peux faire appel à une mesure possible. Mais... du monde spectroscopique, on ne peut éliminer l'observateur. Exemple: pour mesurer la position d'un électron, il faut au moins un photon qui finit par entrer dans l'oeil. Ceci cause un effet considérable surl'électron. Donc, on ne peut pas dire que l'électron, après avoir été observé, se comporte comme si l'observation n'avait pas eu lieu».

Le grand physicien-philosophe John Wheeler nous résume le sens de l'effet de la mécanique quantique sur notre perception de l'univers ainsi: "Aucune description d'un phénomène naturel dans le domaine atomique n'est possible sans faire référence à l'observateur, non seulement quant à sa vitesse, dans le contexte relativiste, mais quant à toutes ses activités en poursuivant ses observations».

Alors, l'humanité revient davantage à une position d'intérêt et d'influence dans l'univers. 


\subsection{Les sciences neurologiques}

Nous parlons de l'observateur et de son influence sur l'univers. Or, l'observateur perçoit, par une chaîne de mécanismes : les sens, les nerfs, la structure du cerveau, ses neurones et leurs états électriques et énergiques. Les connaissances de ces processus sont très avancées.

Au vingtième siècle, le progrès de ces connaissances a amené des surprises. Nous pensons surtout aux travaux des prix Nobel, Sir John Eccles et Roger Sperry, ainsi qu'à l'œuvre du canadien Wilder Penfield à Montréal.

La découverte la plus surprenante est sans doute celle que la conscience (ou l'intelligence) de l'homme ne réside pas dans la structure matérielle du cerveau.

Bien sûr, la conscience utilise le cerveau comme instrument privilégié de sa volonté, mais cette dernière ne réside pas dans la structure atomique des neurones. Feynman nous donne un bel exemple de la signification: "Je me promène sur la plage en regardant les vagues. Les photons reflétés de leur surface pénètrent dans mes yeux et les signaux électriques résultants viennent jusqu'à mes neurones et conditionnent les atomes qui s'y trouvent. Un an plus tard, je reviens, je regarde les vagues et je me souviens de les avoir vues auparavant. Mais, nous savons que les atomes du cerveau sont passagers; les études avec le phosphore radioactif démontrent que la vie moyenne de ces atomes dans le cerveau est de huir jours. Alors les atomes sensibilisés par les photons parvenant des vagues sont depuis longtemps disparus alors que, l'année suivante, je me souviens d'avoir vu les vagues. Alors qu'est-ce qui se souvient? Est-ce la position des aromes remplaçants? La mémoire, est-ce de la géomérrie, i.e. non matérielle? »

Penfield a été passablement surpris de la découverte. Il écrit : "Ma carrière scientifique durant, j'ai essayé, comme plusieurs autres, de démontrer que le cerveau est le site de la conscience. Comme il est admirable de découvrir que le scientifique, lui aussi peut croire à l'esprit».

Sir John Eccles, pour sa part, nous dit : «Il n'y a, alors, aucune évidence scientifique qui peut nier la liberté de la volontébumaine, 
que nous devons postuler, ironiquement, si nous agissons comme des chercheurs scientifiques".

Dans un discours à Edmonton, en avril 1988, M. Michel Gervais, Recteur de l'Université Laval, dit : «Parler de la connaissance comme une fin en soi et de la recherche comme répondant à une nécessité quasi-biologique, c'est faire ressortir l'aspect le plus profond de la valeur de la recherche, mais en même temps celui qui est peut-être le plus méconnu et le plus mis en veilleuse aujourd'bui. Car, autant l'on valorise les fruits de la recherche et ses retombées de tous ordres, autant l'on semble méconnaître la nature de la recherche elle-même en ce qui constitue le motif profond».

Ce train de pensée a ouvert le débat sur des horizons plus larges. Dans une lettre à Maurice Solovine, Albert Einstein écrit : "Je n'ai pas trouvé de meilleure expression que "religieux" pour exprimer la confiance dans la nature rationnelle de la réalité».

En somme, dans la science neurologique du $20^{\text {e }}$ siècle, nous découvrons que la conscience de l'humaniré - l'intelligence humaine - n'est pas matérielle, même si ses décisions affectent l'univers dans son évolution, surtout au niveau atomique.

\subsection{La cosmologie}

Les investigations de l'astronomie du $20^{e}$ siècle nous démontrent que les galaxies qui composent l'univers sont en fuite mutuelle et que les plus distantes s'éloignent davantage avec des vitesses correspondantes à leurs distances. Nous avons alors le portrait d'un univers en explosion, dont les parties les plus rapides sont naturellement les plus distantes. Cette vision des choses, élaborée par l'abbé Georges-Henri Lemaître et George Gamow, implique un instant d'explosion, un début de l'univers. Ce moment est maintenant fixé à il y a environ 15 milliards d'années.

Les conditions qui ont prévalu à cet instant du début ont occasionné la formation des nucléons, des électrons et d'autres composantes du monde, et ont guidé leur évolution en galaxies et en étoiles, où se sont développés les éléments lourds qui ont comme résultat la vie biologique et sa progression par sélection naturelle sur des planètes comme la Terre. 
Maintes vérifications expérimentales sur l'abondance et la distribution des éléments dans les étoiles, sur leurs spectres et leur luminosiré, sur leur progression d'un amas de gaz vers des supernovae, et ainsi de suite, ont confirmé la réalité de l'hypothèse du début discontinu de l'univers.

À cette vérification, Rasetti a également contribué. Ses études spectroscopiques, d'abord, ont ajouté au catalogue des données composant l'édifice de la théorie. Aussi, avec Paul Koenig, ses recherches sur les débris cosmiques et les porteurs de l'énergie de liaison nucléaire que sont les mesons ont aidé à construire plus précisément notre description de l'ensemble.

L'idée de l'univers immuable, issu de la description mécanique ne s'est pas effacée sans efforts pour la maintenir. Sir Fred Hoyle proposa un univers stable, maintenu en expansion par la création continue d'une petite quantité d'hydrogène. Cette suggestion de la création continue s'est heurtée à la découverte de la radiation résiduelle de l'explosion primitive, qui donne à l'univers courant une température de $3^{\circ} \mathrm{K}$. Puis, l'on a suggéré un univers éternel mais oscillant, où une expansion comme l'actuelle serait suivie d'une contraction vers un point, puis de nouveau une explosion, et ainsi de suite ad infinitum. Weinberg a démontré qu'un tel univers serait caractérisé par une augmentation de l'entropie qui changerait à chaque cycle le rapport des photons sur les nucléons. Dans un univers éternel, ce rapport serait infini. Or, il ne l'est pas.

Nous sommes alors dans un univers qui a débuté, qui n’est pas éternel et qui évolue.

Il a été remarqué que toutes les conditions dans cet univers à travers 15 milliards d'années, et surtout durant les premières millisecondes, ont été critiques pour l'évolution de la vie. Les paramètres - masse du proton, charge de l'électron, ampleur des forces - en ne variant que d'une quantité très faible, auraient rendu la vie impossible. Les marges sont très restreintes pour la vie humaine en particulier. Le nombre des coïncidences propices à l'existence de la vie est tellement élevé qu'il semble que l'univers aurait été dessiné pour nous accommoder. Le grand cosmologue de notre temps, Stephen Hawking, écrit : «Nous sommes poussés à la conclusion que l'isotropie de l'univers et notre existence résultent du fait que l'univers est en expansion à un taux critique. Étant 
donné que nous ne pourrions observer l'univers comme étant différent si nous n'y étions pas, nous pouvons dire que l'isotropie de l'univers est une conséquence de notre existence».

Notez bien: "l'isotropie de l'univers est une conséquence de notre existence », pas l'inverse. C'est une des idées les plus difficiles pour les matérialistes à accepter.

John Wheeler le dit plus succinctement: "L'univers a les dimensions qu'il a parce que nous y sommes".

Ce point de vue que l'univers est destiné pour l'humanité constitue une version du principe anthropique. C'est un principe qui a été en vogue maintes fois dans l'histoire de la science et de la philosophie. Couramment il attire beaucoup d'attention, et nombreux sont les livres et les articles en traitant qui arrivent dans nos bibliothèques depuis cinq ans.

Cette description, issue de la cosmologie, remplace le modèle d'un univers éternel et mécanique par un modèle dynamique, ayant un début et des caractéristiques intentionnelles pour l'humanité.

\section{RÉSUMÉ}

Résumons cette perception rapide de certains événements intellectuels importants du $20^{\mathrm{e}}$ siècle.

À travers l'histoire, les idées scientifiques ont beaucoup contribué à l'évolution de la philosophie. En particulier, la théorie mécanique, initiée vers l'époque de Copernic et mise au point par Newton a donné lieu à la philosophie matérialiste des trois derniers siècles. Cette philosophie nous présente un monde absolu et déterminé, dans lequel l'humanité occupe une place négligeable et qui n'a pas de place pour les qualités abstraites comme la beauté, la compassion, la volonté libre. Dieu n'est pas nécessaire à cette philosophie.

Depuis, des avancées en relativité, en mécanique quantique, dans les sciences neurologiques, en astronomie et en cosmologie nous permettent une perception d'un monde plus réel. Nous pouvons maintenant comprendre que l'univers est temporel, dynamique, intentionnel pour l'humanité et influencé par des 
agents non matériels. Cette perception initie alors une nouvelle philosophie humaniste, comprenant le principe anthropique dont le sort a beaucoup varié à travers les âges.

Les philosophes matérialistes sont alors perçus comme constituant un hiatus de trois siècles dans l'évolution de la pensée humaine.

La nouvelle philosophie met de côté les notions sombres et pessimistes de la philosophie matérielle. Elle fait place au non matériel : l'esprit, la conscience, la beauté, le spirituel, les vertus. Du point de vue de la science, tout cela me paraît plus simple et plus élégant, donc préférable.

Mais est-ce vrai? Durera-t-elle?

Comme l'a dit Churchill au sujet d'Arthur, il est tout vrai, ou il devrait l'être.

Quant à la durée, il est inévitable que l'évolution de la pensée humaine continuera et que d'autres philosophies se succèderont. De nos jours, une autre vague d'idées nouvelles plane sur la science. Nous parlons de super-accélérateurs, de supraconductivité, de super-ficelles. Y sera-t-il peut-être question d'une philosophie super humaine? Sans doute.

Pour un scientifique, il est plus qu'intéressant de suivre l'influence que sa discipline peut avoir sur les thèses des grands intellectuels et je suis heureux d'avoir vécu durant un siècle si prometteur. Heureux surtout d'avoir pu connaître l'un des grands à apporter une contribution aux idées fondamentales qui nous animent aujourd'hui, celui que nous honorons, le professeurdocteur Franco Rasetti.

\section{Président}

Agence spatiale canadienne 